\title{
Detection of tiny amounts of fissile materials in large-sized containers with radioactive waste
}

\author{
V.F. Batyaev, S.V. Skliarov \\ Dukhov Research Institute of Automatics (VNIIA), 127055 Moscow, Russia \\ bogolubov@vniia.ru
}

\begin{abstract}
The paper is devoted to non-destructive control of tiny amounts of fissile materials in large-sized containers filled with radioactive waste (RAW). The aim of this work is to model an active neutron interrogation facility for detection of fissile materials inside NZK type containers with RAW and determine the minimal detectable mass of $\mathrm{U}-235$ as a function of various parameters: matrix type, nonuniformity of container filling, neutron generator parameters (flux, pulse frequency, pulse duration), measurement time. As a result the dependence of minimal detectable mass on fissile materials location inside container is shown. Nonuniformity of the thermal neutron flux inside a container is the main reason of the space-heterogeneity of minimal detectable mass inside a large-sized container. Our experiments with tiny amounts of uranium-235 $(<1 \mathrm{~g})$ confirm the detection of fissile materials in NZK containers by using active neutron interrogation technique.
\end{abstract}

Index Terms-Fissile materials, Neutron generator, MonteCarlo simulating

\section{INTRODUCTION}

One of the urgent challenges of today's nuclear power engineering is to detect and control fissile material (FM) in various radioactive waste (RAW). The expediency of its solution lies both in ensuring long-term nuclear and radiation safety of RW storage sites, and in preventing theft of FM.

The major part (at least 99\%) of RAW in Russia belong to low and very low level wastes (LLW) [1], which, as a rule, are packed into the NZK containers [2], the FM content there being estimated in the range $\sim 10^{-2}$ to $\sim 10 \mathrm{mg} / \mathrm{kg}$ of LLW [3]. The legislation of the Russian Federation obliges the certification procedure of RAW packages before their disposal, in particular, for the presence of FM. The opening of the container for such certification is not permitted.

It is clear that the existence of fission products, as well as the neutron emitting actinides in the certified RAW packages leads to increased the gamma and/or neutron background around the containers and the deterioration of FM control. Nevertheless, at the first stage of designing the required certifier, our theoretical calculations were made under the assumption that only uranium isotopes and no fission products and other actinides were present in the NZK. The expediency of this assumption is supported by the fact that part of the nuclear fuel cycle facilities produce RAW containing uranium isotopes only.

Previously, we carried out a calculation work [4] comparing non-destructive methods for controlling small amounts of FM in large-sized containers with radioactive waste, in order to select the method and determine the ways to implement it. It was shown that the use of the passive gamma-neutron control method is difficult due to the small amount of FM in containers and the high absorbing capability of both the container itself and the filler matrix. Executed evaluations of FM responses to the effect of neutron (2.5 MeV and $14 \mathrm{MeV})$ and gamma sources $(10 \mathrm{MeV})$ on the container show the advantage of active neutron methods for controlling large containers in front of the photonuclear method in terms of the absolute values of response radiation ensuring control of these small amounts of FM in the NZK.

This work consists in modeling the active neutron control system for FM in the NZK containing LLW and determining the minimum detectable mass of FM depending on various factors (measurement duration, number of neutron detectors, type of matrix and uneven distribution of FM in the NZK), and also in improving the proposed method.

\section{MODELING OF ACTIVE NEUTRON CONTROL SYSTEM FOR DETECTION OF FM IN NZK CONTAINING LLW}

The first step is to determine the sensitivity of the neutronradiation method, depending on the position of uranium-235 in various zones of the NZK. For this purpose, a mathematical model of the container and its contents was prepared for computational modeling of neutrons transport and interaction.

The container is a hollow concrete cube with external dimensions of $165 \mathrm{~cm} \times 165 \mathrm{~cm} \times 134 \mathrm{~cm}$, a wall thickness of $11 \mathrm{~cm}$, a weight of 3.5 tons, which is filled with $\sim 3.2$ tons of concrete matrix containing LLW.

As the material of the walls, reinforced concrete with a density of $2.5 \mathrm{~g} / \mathrm{cm} 3$ was selected, while FM was placed in the concrete matrix of the container. The container matrix was split into 25 zones (Figure 1), with FM (uranium-235) sequentially located in the middle of each of the zones of the NZK over the height of the zone. As neutron detectors, SNM-18 3-He-counters were simulated, surrounded by polyethylene and a cadmium shield. The source of neutrons was chosen as a pulsed point isotropic one with $14 \mathrm{MeV}$ energy. An example of the obtained time distributions is shown in Fig. 2, which shows that the first exponent corresponds to the detection of initial fast neutrons and its decay constant is determined by the thickness inner moderator inside the cadmium shield of the counter. The second exponent corresponds to the thermalized neutrons (background) and fast neutrons of FM fission. The decay constant of the second exponential is determined by the walls of the NZK and its filling. 


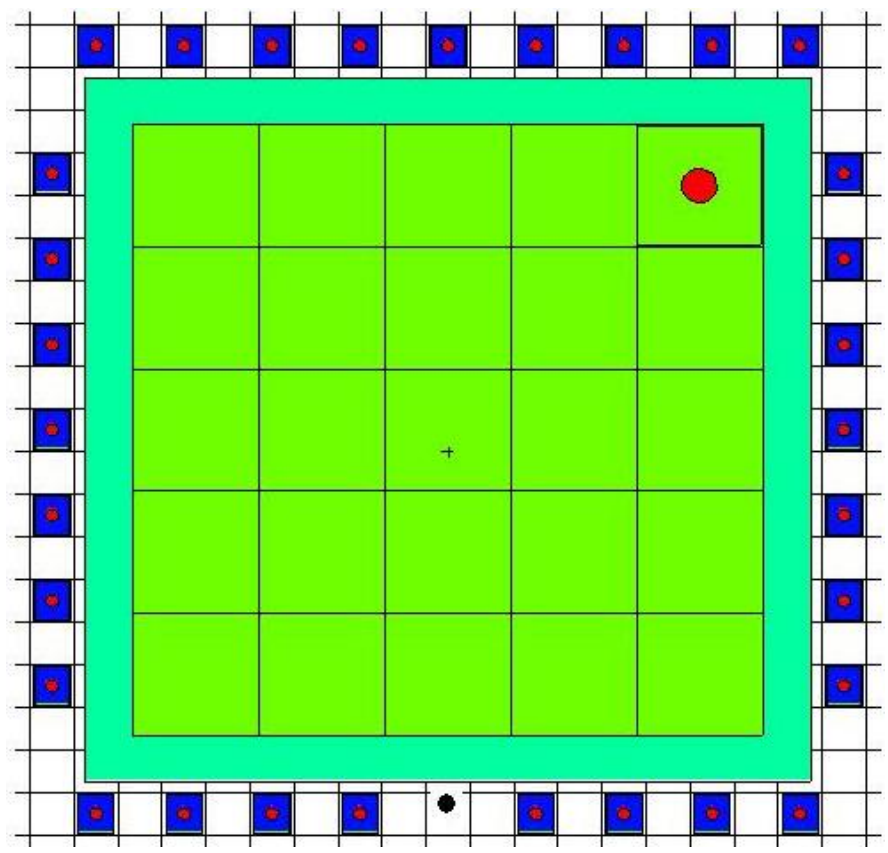

Fig. 1 Calculation model of NZK with location of FM in one of container zones. The dot shows the position of the neutron source (14 MeV neutron generator ING-07T produced at VNIIA).

The simulation results allowed to determine the minimum detectable mass of FM as a function of its position in the NZK container as well as its dependence on the number of neutron counters used (Figures 3 and 4), provided that their backgrounds are the same. The wide range of the minimum detectable mass of uranium-235 (from 2 to $84 \mathrm{mg}$ ) shown in Figures 3 and 4 is due to the non-homogeneity of the thermal neutron field inside the container, which in practice can be partially neutralized by rotating the container during the measurement.

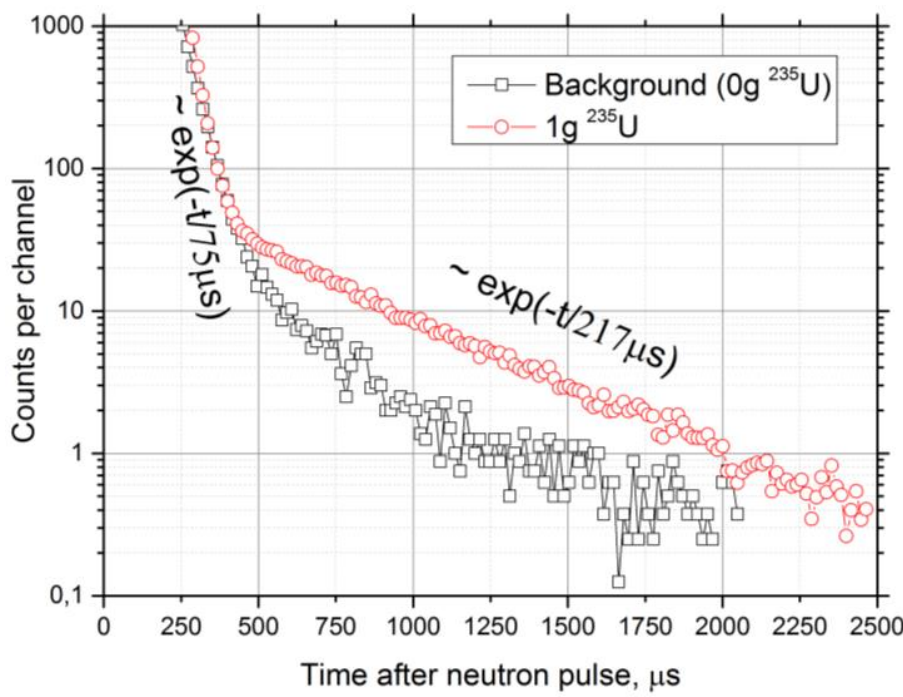

Fig. 2 - Time distributions of counting rate of neutron counter closest to ING after neutron pulse. The flux of ING$07 \mathrm{~T}$ is $5 \times 10^{8} \mathrm{n} / \mathrm{s}$, measurement duration is 8 minutes.

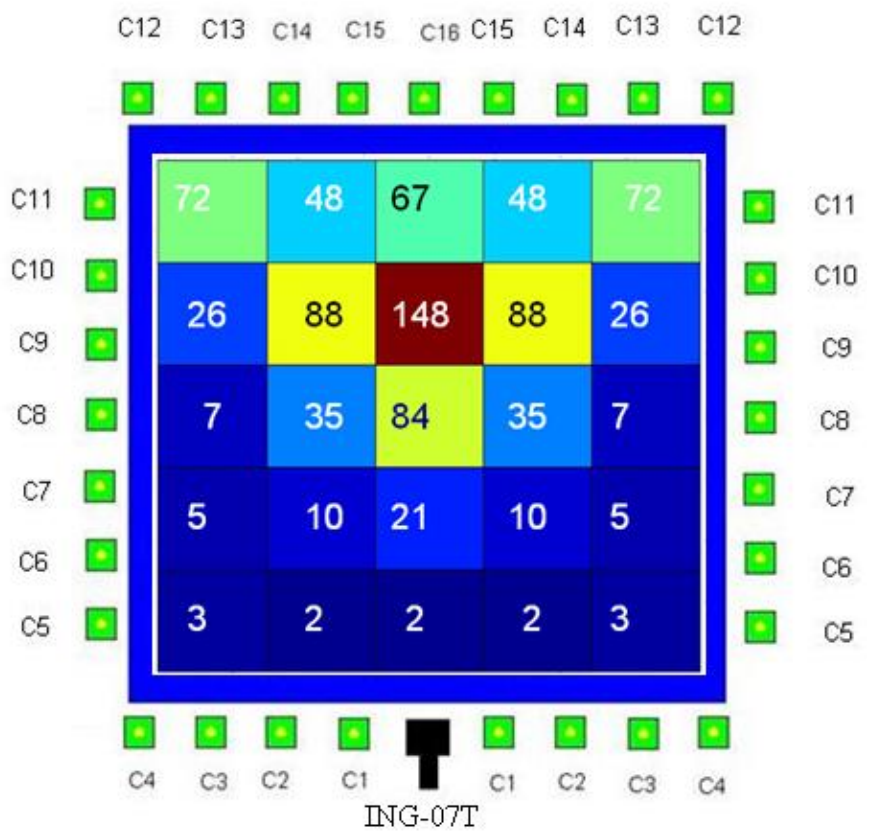

Fig. 3 - Simulated minimum detectable mass of ${ }^{235} \mathrm{U}$ (mg) depending on its location in container.

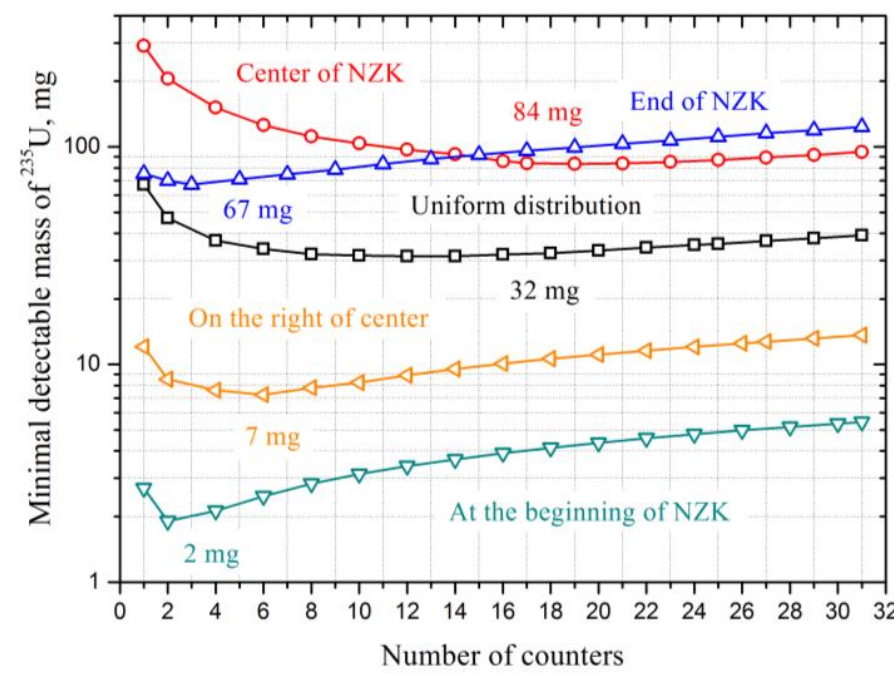

Fig. 4 - Simulated minimum detectable mass of ${ }^{235} U$ (mg) depending on FM location in container and number of counters used in analysis.

\section{EXTERNAL SHIELDING OF NEUTRON COUNTERS}

The above simulation studies were made without taking into account the materials surrounding the system (floor, walls, ceiling). Therefore, the second stage was to determine their effect primarily on the background sensitivity of neutron counters. For this purpose, calculations were made of the time spectra of background neutrons reflected from surrounding walls, located $3 \mathrm{~m}$ from the system (Fig. 5).

Fig. 5 shows that in the time range of interest of 500-2,000 $\mu$ s after the neutron pulse, the neutron detectors will be exposed to reflected neutrons with energy from thermal to several dozen $\mathrm{eV}$. Neutrons with energy below $\sim 0.5 \mathrm{eV}$ can be absorbed by 
the cadmium shield of the counters, while neutrons with high energies (epithermal) can be detected, thus making an unnecessary contribution to the background.

To minimize this effect, it was proposed to cover the neutron counters with a neutron-absorbing external shield. The calculated time distribution of the count rate of a neutron detector with and without a shield in the form of borated polyethylene are shown in Fig. 6. In addition, we carried out the corresponding experiments, which confirmed the calculated results.

Application of these shields should ensure that the minimum detectable mass of FM estimated in the previous paragraph is preserved.

\section{CONCLUSION}

The simulated and experimental results of detecting of small amounts of FM in large-sized NZK containers are presented. Executed estimates of the minimum detectable mass, depending on the position of FM in the NZK, show a strong scatter of its magnitude (from units to dozens of $\mathrm{mg}$ ), which is due to the inhomogeneity of the thermal neutron field inside the NZKMR.

The conducted experiments on detection of FM behind concrete shielding showed an unambiguous possibility of detecting small amounts of FM (dozens of mg) in the NZK.

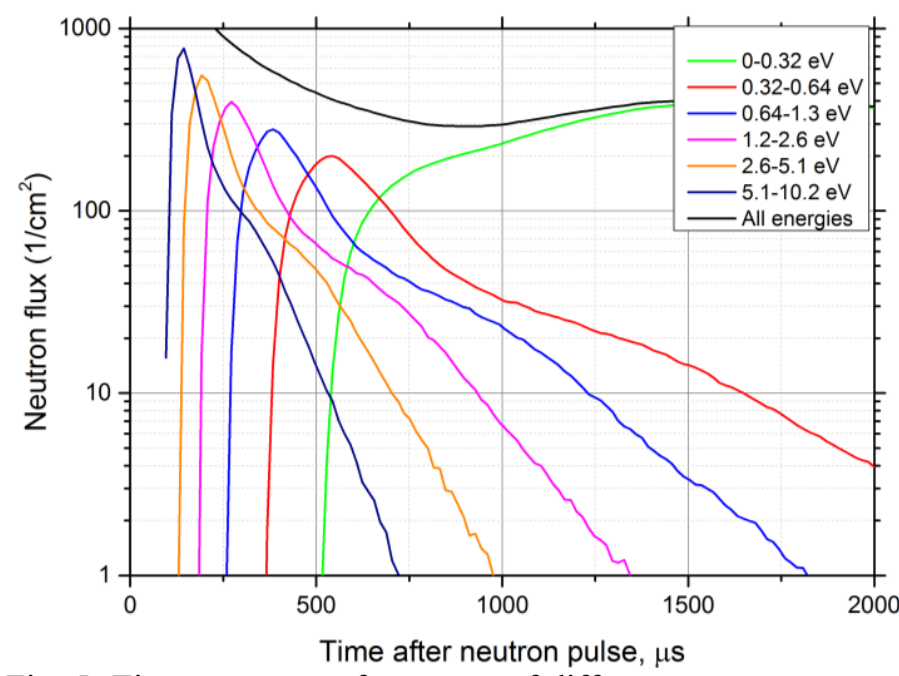

Fig. 5. Time spectrum of neutrons of different energy groups reflected from concrete wall $3 \mathrm{~m}$ apart from 3 -He counters

It was demonstrated that the background level of the reflected neutrons can make a decisive contribution into the value of the minimum detectable mass of FM, which requires appropriate shielding of neutron detectors from epithermal neutrons.

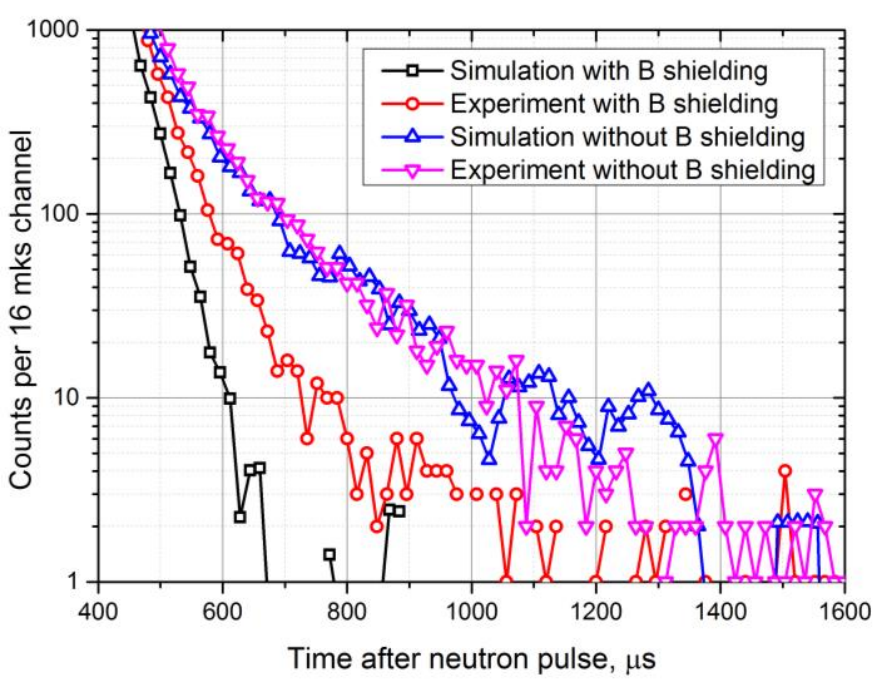

Fig. 6. Computational modeling of neutron detectors shielding with $8 \%$ borated polyethylene of $3 \mathrm{~cm}$ thickness.

\section{REFERENCES}

[1] www.norao.ru/waste/where-is/

[2] NZK-MR concrete container, http://www.radon.ru/eng/activities/radwaste management/conditioning/

[3] V.F. Batyaev, S.V. Sklyarov, Potential of active neutron interrogation to control fissile materials in "closed" fuel cycle, IAEA conference, Wien 2015

[4] V.F. Batyaev, S.V. Sklyarov, Comparative Analysis of Non-destructive Methods to Control Fissile Materials in Large-size Containers. International Conference Nuclear Data 2016, Bruges, Belgium, 2016 